\title{
Matrix Metalloproteinase 28-Bearing Microcystic Adnexal Carcinoma on the Back
}

\author{
Hiromu Chiba Yumi Kambayashi Erika Tamabuchi Akira Hashimoto \\ Taku Fujimura \\ Department of Dermatology, Tohoku University Graduate School of Medicine, Sendai, \\ Japan
}

\section{Keywords}

Microcystic adnexal carcinoma - Radiation resistant - Matrix metalloproteinase $28 \cdot$ Nerve infiltration - Local recurrence

\section{Abstract}

Although microcystic adnexal carcinoma (MAC) recurs and expands locally without metastases to the lymph nodes and other organs, its biological behavior remains unknown. In this report, a case of a giant MAC on the back treated with radical excision and curative radiation therapy and its characteristic dermoscopic findings are presented. In addition, immunohistochemical staining showed the expression of matrix metalloproteinase (MMP) 28 on the tumor cells and MMP12 and periostin in the stroma. The present case suggests the possible biological behaviors of MAC and might provide a possible target for the treatment of MAC in the future.

\section{Introduction}

Microcystic adnexal carcinoma (MAC) is an indolent but deeply infiltrative tumor with a high propensity for perineural invasion [1]. MAC characteristically recurs and expands locally without metastases to the lymph nodes and other organs $[1,2]$. Since 


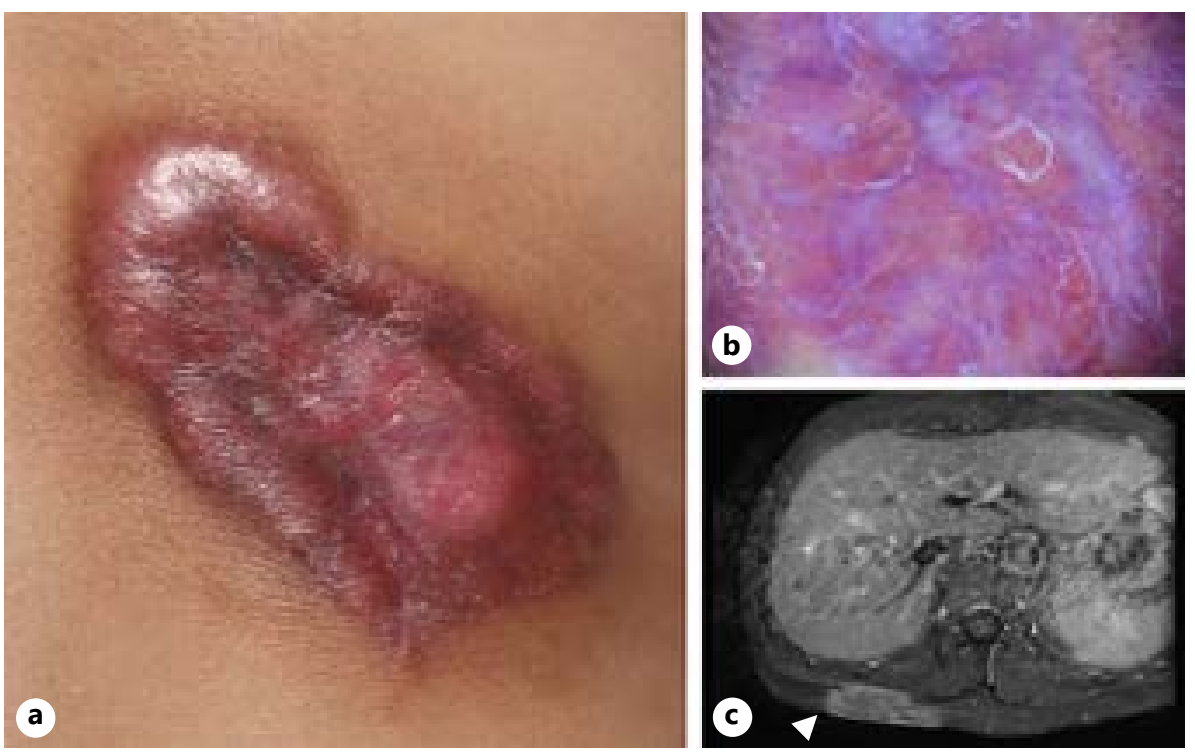

Fig. 1. a An infiltrated red tumor on the back. b Dermoscopic findings of the tumor: whitish mesh-like structure with arborizing vessels.

MAC can extend far beyond the clinical margin, its treatment is challenging [1-3]. In this report, a case of MMP28-expressing MAC on the back with MMP12 and periostin (POSTN) in the cancer stroma is presented.

\section{Case Report}

A 55-year-old Japanese woman visited our outpatient clinic with an infiltrated, easy-tobleed tumor on her back. She was aware of her tumor 30 years earlier. On her initial visit, physical examination showed an infiltrated red tumor, $10 \mathrm{~cm} \times 5.5 \mathrm{~cm}$ in size, on the back (Fig. 1a). Dermoscopy showed a whitish mesh-like structure with arborizing vessels (Fig. 1b). MRI findings suggested tumor infiltrating into the latissimus dorsi (Fig. 1c). A biopsy specimen showed numerous keratinous cysts and small islands of basaloid and squamous epithelium with ductal differentiation throughout the superficial to deep dermis (Fig. 2a). In addition, smaller nests and strands of cells in a desmoplastic stroma (Fig. 2b) with nerve invasion (Fig. 2c, d) were prominent in the deep dermis. On immunohistochemical staining, these tumor cells were positive for AE1/AE3 and negative for CEA and BerEP4. From the above findings, the diagnosis was MAC on the back with nerve invasion. The tumor was excised with a 30-mm margin and part of the latissimus dorsi and trapezius muscles. In addition to surgical treatment, intensity-modulated radiotherapy (60 Gy: $2.0 \mathrm{~Gy} \times 30$ fractions) was given as adjuvant therapy. Nine months after intensive treatment, her tumor was still under control.

Since the dermoscopic and histological finding suggested fibrosis and vascularization of the tumor, to further investigate the possible biological behaviors of MAC, immunohistochemical staining for MMP12, MMP28, and POSTN, which correlate with angiogenesis and fibrosis of skin tumors, was performed. The MMP12 expression (Fig. 3a) was weakly positive at stroma compared to positive (Fig. 3b) and negative (Fig. 3c) control. POSTN (Fig. 3d) was highly expressed in the cancer stroma that showed the desmoplastic findings of MAC, whereas MMP28 (Fig. 3g) was expressed on tumor cells. In addition, positive and negative controls for POSTN (Fig. 3e, f) and MMP28 (Fig. 3h, i) were presented.

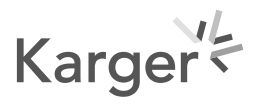




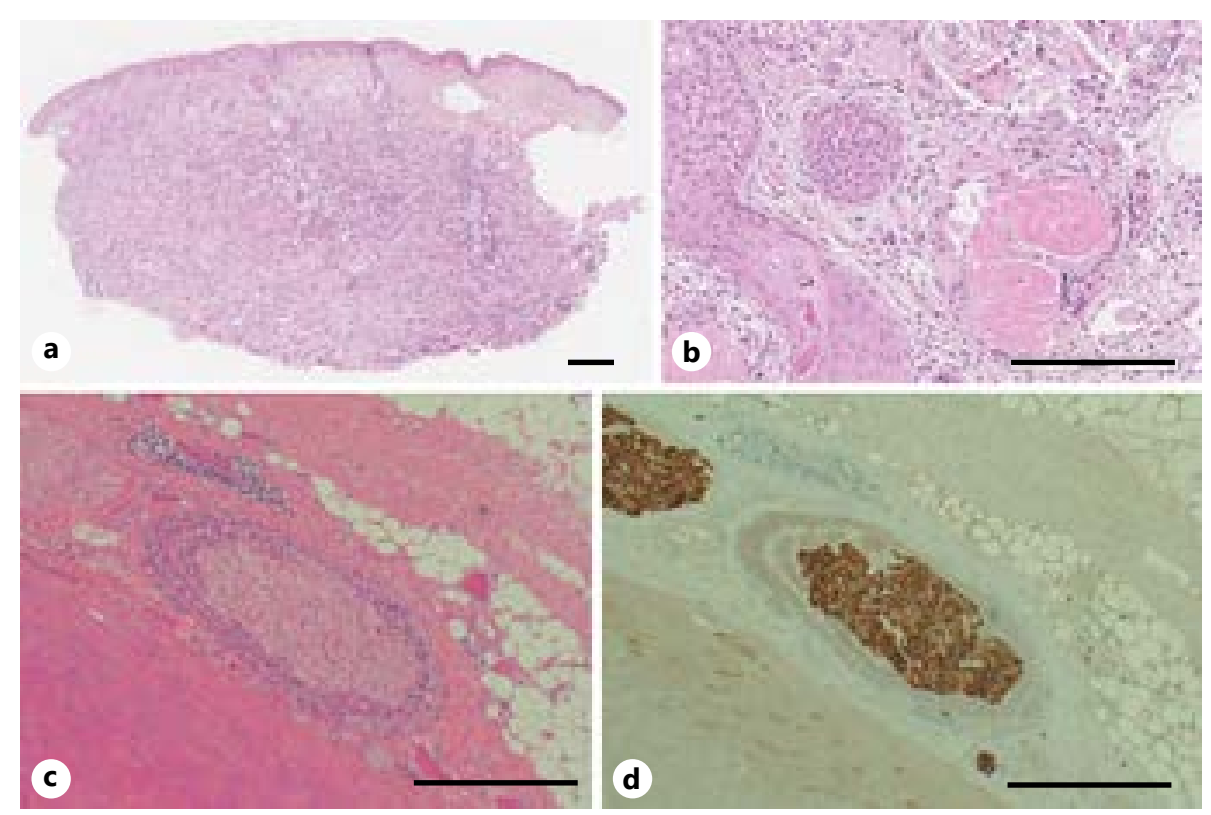

Fig. 2. a Numerous keratinous cysts and small islands of basaloid and squamous epithelium with ductal differentiation throughout the superficial to deep dermis. In addition, smaller nests and strands of cells in a desmoplastic stroma (b) with nerve invasion (c, d) are prominent in the deep dermis (hematoxylin and eosin staining (a-c), S100 staining (d)) (scale bar, $200 \mu \mathrm{m}$ (a) and $100 \mu \mathrm{m}$ (b-d)).

\section{Discussion}

MAC, also known as sclerosing sweat duct carcinoma, is a rare malignant cutaneous tumor of the skin with pilar and eccrine differentiation [6]. It is a locally aggressive, indolent, but infiltrative tumor, potentially invasive from dermis to muscle, adipose tissue, and nerves [6]. The characteristic histological features of MAC are small keratin-filled cysts, islands of basaloid keratinocytes, and ductal structure surrounded by desmoplastic stroma [6]. In addition, the dermoscopic findings in the present case showed a prominent whitish mesh-like structure with arborizing vessels, suggesting fibrosis and vascularization of the tumor. From the above findings, it was hypothesized that several MMPs might play roles in characterizing of biological behavior of MAC.

MMPs play significant roles in cancer formation. Among them, MMP12 is a potent protease that inhibits tumor angiogenesis and metastasis by catalyzing the formation of antiangiogenic peptides [7]. In skin cancer, decreased levels of MMP12 could be correlated with vascularization, angiogenesis, and tumor invasion of SCC and EMPD [4]. MMP28 plays a role in remodeling the newly formed basement membrane during wound repair [8] and is even highly expressed in sclerosing diseases such as limited-type scleroderma [9]. In addition to MMP28, POSTN is a matrix cellular protein that induces the production of pro-inflammatory cytokines such as IL-1 $\beta$ and TNF- $\alpha$ from fibroblasts, leading to the development of fibrosing diseases [10]. Furthermore, POSTN promotes the production of MMP12 from M2 macrophages [11]. Since MAC is abundant in the desmoplastic area and the highly vascularized skin tumor that infiltrates deeply into the dermis, these MMPs and POSTN might be highly expressed by MAC. In fact, in the present case, the cancer stroma possessed POSTN and MAC cells expressed MMP28, but density of MMP12 in cancer stroma was relatively low compared to basal cell carcinoma. Since MAC can extend far beyond the clinical margin and can recur years after initial treatment, its treatment is challenging [1]. Although a previous report suggested the efficacy of radiotherapy for MAC [12], in most cases, chemotherapy was ineffective [13].

\section{Karger's}




\section{Case Reports in Oncology}
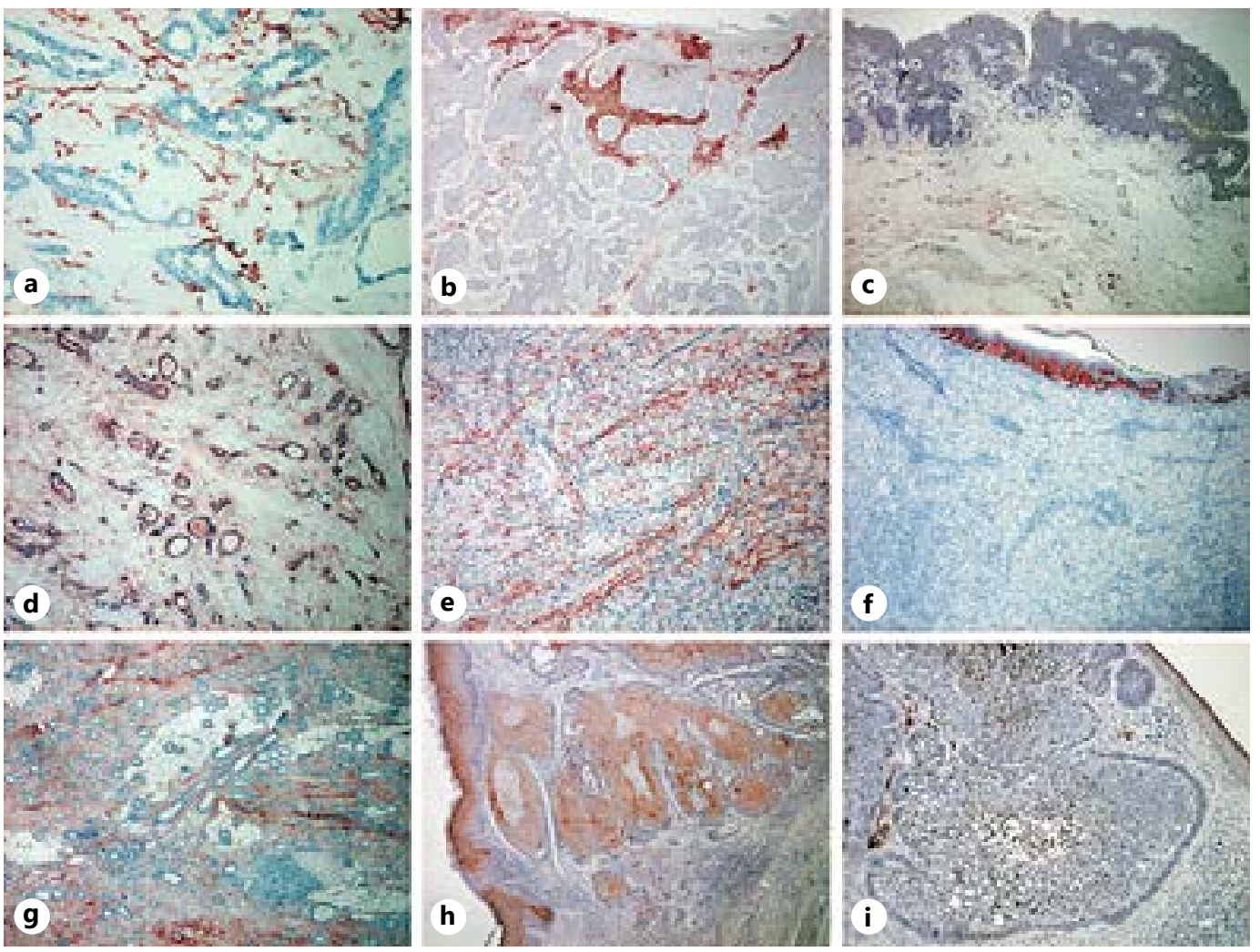

Fig. 3. Paraffin-embedded tissue samples were deparaffinized and stained with anti-MMP12 Ab (a-c), antiPOSTN Ab (d-f), and anti-MMP28 Ab (g-i) (scale bar, $100 \mu \mathrm{m}(\mathbf{a}-\mathbf{i})$ ). Positive and negative controls were selected as we previously reported [4,5]. Present case (a, d, g), positive control (basal cell carcinoma (b), dermatofibroma (e), and squamous cell carcinoma (h)), and negative control (extramammary Paget's disease (c), dermatofibrosarcoma protuberans (f), and basal cell carcinoma (i)).

Therefore, additional treatments for MAC are needed. Notably, several drugs such as bisphosphonates, statins, and tetracyclines have already been reported to modulate the production of MMPs and even to improve the prognosis of cancer patients $[14,15]$. Indeed, previous reports suggested that bisphosphonates might enhance the therapeutic effect of docetaxel in cutaneous angiosarcoma $[16,17]$. These reports suggest the potential benefit of bisphosphonates, statins, and tetracyclines to improve the prognosis of MMPs-bearing MACs.

\section{Conclusion}

The present case suggests the possible biological behaviors of MAC and might provide a possible target for the treatment of MAC in the future.

\section{Statement of Ethics}

Written informed consent was obtained from the patient for publication of this case report and any accompanying images. The protocol for this human study was approved by the Ethics Committee of the Tohoku University Graduate School of Medicine, Sendai, Japan (Permit No. 23597). 


\section{Conflict of Interest Statement}

The authors have no conflicts of interest to declare.

\section{Funding Sources}

This study was supported in part by the Japan Agency for Medical Research and Development (21ym0126041h0001).

\section{Author Contributions}

Fujimura T. designed the research study. Chiba H., Kambayashi Y., Tamabuchi E., Hasimoto A., and Fujimura T. treated the patient and acquired the clinical data. Chiba H. and Fujimura T. wrote the manuscript. Fujimura T. supervised the study.

\section{Data Availability Statement}

Data sharing is not applicable to this article as no datasets were generated or analyzed during the current study.

\section{References}

1 Gordon S, Fischer C, Martin A, Rosman IS, Council ML. Microcystic adnexal carcinoma: a review of the literature. Dermatol Surg. 2017;43:1012-6.

2 Chaudhari S, Blanchard L, Chaudhari PJ, Lythgoe T. The first reported case of metastatic microcystic adnexal carcinoma in an adolescent. Dermatol Surg. 2015;41:286-8.

3 Haga T, Fujimura T, Aiba S. Successful treatment of microcystic adnexal carcinoma on the philtrum with oral S-1 monotherapy. J Dermatol. 2019;46:e208-9.

4 Fujimura T, Okabe T, Tanita K, Sato Y, Lyu C, Kambayashi Y, et al. A novel technique to diagnose non-melanoma skin cancer by thermal conductivity measurements: correlations with cancer stromal factors. Exp Dermatol. 2019;28:1029-35.

5 Fujimura T, Kakizaki A, Sato Y, Tanita K, Furudate S, Aiba S. The immunological roles of periostin/tumor-associated macrophage axis in development of dermatofibrosarcoma protuberans. Anticancer Res. 2017;37: 2867-73.

6 Aslam A. Microcystic adnexal carcinoma and a summary of other rare malignant adnexal tumours. Curr Treat Options Oncol. 2017;18(8):49.

7 Dandachi N, Kelly NJ, Wood JP, Burton CL, Radder JE, Leme AS, et al. Macrophage elastase induces TRAILmediated tumor cell death through its carboxy-terminal domain. Am J Respir Crit Care Med. 2017;196:353-63.

8 Illman SA, Lohi J, Keski-Oja J. Epilysin (MMP-28) - structure, expression and potential functions. Exp Dermatol. 2008;17:897-907.

9 Kakizaki A, Fujimura T, Furudate S, Kambayashi Y, Aiba S. Immunohistochemical similarities between lichen sclerosus et atrophicus and morphea: a case study. Case Rep Dermatol. 2015;7:39-45.

10 Uchida M, Shiraishi H, Ohta S, Arima K, Taniguchi K, Suzuki S, et al. Periostin, a matricellular protein, plays a role in the induction of chemokines in pulmonary fibrosis. Am J Respir Cell Mol Biol. 2012;46:677-86.

11 Furudate S, Fujimura T, Kakizaki A, Kambayashi Y, Asano M, Watabe A, et al. The possible interaction between periostin expressed by cancer stroma and tumor-associated macrophages in developing mycosis fungoides. Exp Dermatol. 2016;25:107-12.

12 Pugh TJ, Lee NY, Pacheco T, Raben D. Microcystic adnexal carcinoma of the face treated with radiation therapy: a case report and review of the literature. Head Neck. 2012;34:1045-50.

13 Chaudhari SP, Mortazie MB, Blattner CM, Garelik J, Wolff M, Daulat J, et al. Treatments for microcystic adnexal carcinoma - a review. J Dermatolog Treat. 2016;27:278-84.

14 Choi J, Lee EJ, Yang SH, Im YR, Seong J. A prospective phase II study for the efficacy of radiotherapy in combination with zoledronic acid in treating painful bone metastases from gastrointestinal cancers. J Radiat Res. 2019;60:242-8. 
15 Sarker H, Haimour A, Toor R, Fernandez-Patron C. The emerging role of epigenetic mechanisms in the causation of aberrant MMP activity during human pathologies and the use of medicinal drugs. Biomolecules. 2021;11(4):578.

16 Kambayashi Y, Fujimura T, Furudate S, Hashimoto A, Haga T, Aiba S. Comparison of immunosuppressive and cytotoxic cells in angiosarcoma: development of a possible supportive therapy for angiosarcoma. Dermatology. 2013;227:14-20.

17 Ishibashi M, Fujimura T, Hashimoto A, Haga T, Onami K, Tsukada A, et al. Successful treatment of MMP9-expressing angiosarcoma with low-dose docetaxel and bisphosphonate. Case Rep Dermatol. 2012;4:5-9. 